\title{
Improved Angle Diversity Non-imaging Receiver with a Help of Mirror in Indoor MIMO-VLC Systems
}

\author{
Ki-Hong Park and Mohamed-Slim Alouini \\ Computer, Electrical, Mathematical Sciences and Engineering (CEMSE) Division \\ King Abdullah University of Science and Technology (KAUST) \\ Thuwal, Kingdom of Saudi Arabia \\ \{kihong.park; slim.alouini\}@kaust.edu.sa
}

\begin{abstract}
In this paper, we propose the new design of pyramidal angle diversity receiver with a help of mirror in indoor multiple-input multiple output (MIMO) visible light communication (VLC) systems. Embedding the mirror between the photodetector (PD) planes can help compensate for the high correlation of MIMO-VLC channel matrix. The channel gain of newly proposed receiver structure is derived based on the geometry of transmitter/receiver and azimuth/elevation angles of PD and mirror. From the selected numerical results, we see that the proposed receiver structure can further improve the channel capacity of MIMO-VLC systems when the receiver is located in the region where the channel correlation can dominantly degrade the channel capacity.
\end{abstract}

\section{INTRODUCTION}

Optical wireless communication is the emerging technology facilitated by the advance in the fabrication and development of solid-state lighting devices, i.e., light emitting diode (LED), laser diode (LD), and photodetector (PD) [1]. The inexpensive, efficient and ubiquitous lighting sources enable to develop indoor visible light communication (VLC) which is used for illumination purpose as well as for data transmission variant using visible light between 400 and $800 \mathrm{THz}$ [2]. Multiple lighting sources embedded in ceilings are the foundation for multiple-input multiple-output VLC (MIMO-VLC) systems [3] and VLC cellular networks [4], [5]. Such systems can provide high data rate through spatial multiplexing scheme which has been indeed demonstrated by experiment [6][8]. Moreover, they can serve simultaneous data transmission from/to multiple users in cellular uplink/downlink VLC networks at the coexistence of conventional radio frequency (RF) communication systems [2]. Despite the advantages in VLC systems using multi-source indoor lighting, the line-ofsight (LoS)-dominant VLC intensity channels are preventing the simultaneous transmission because of the resulting highly correlated channel matrix leading to severe intra-cell and intercell interference.

Advances in receiver structures can help reduce the channel correlation and thus improve the system performance. Using an optical lens in front of PDs is beneficial to project the

The research reported in this publication was supported by funding from King Abdullah University of Science and Technology (KAUST). optical intensity signals onto PD planes with partial and completely separated light images. These imaging receivers integrating covex, hemispherical or fisheye lenses [8]-[10] can lead to a well-conditioned channel matrix at the expense of the limited field-of-view (FoV) and increased size and cost of receiver with additional optics. Non-imaging receiver is an another proposed receiver structure which compensate for the high channel correlation. It is modified to separate the light signals from specific direction for each PD without incorporating an optical lens. Several types of non-imaging receivers have been proposed, e.g., line-blocked receiver, spatiallyseparated receiver [11], angle diversity receiver (ADR) [12], prism array receiver [13], multi-FoV array receiver [14] and mirror diversity receiver [15].

Among them, the ADR is widely used in VLC [12], [16] and indoor localization [17] because of its simplicity and performance. It varies the orientation angles of PDs to receive the incident light from one specific direction, while not to reach from other directions. Recently, we proposed mirror diversity receiver in [15] for $2 \times 2$ MIMO-VLC systems and show that deploying a mirror can further improve the system performance compared with some aforementioned nonimaging receivers. Motivated by these, we propose in this paper the angle diversity receiver with a help of mirror to improve the channel capacity of general MIMO-VLC systems. We first design the structure of mirror-aided angle diversity receiver (MADR) based on the combination of pyramidal deployment of PD planes and one-sided mirrors with different azimuth angles. We derive the channel matrix in indoor MIMO-VLC systems with the proposed MADR. Finally, we evaluate the channel capacity through simulation in typical indoor scenario and show that the proposed MADR can further improve the channel capacity around the region relatively close to the room center.

The remainder of this paper is organized as follows. Section II describes a system model of a MIMO-VLC systems and transmitter/receiver structure. We calculate the channel matrix of the proposed receiver structure in Section III. We provide numerical results in Sections IV to validate the superiority of our proposed receiver and finally conclude this work in 


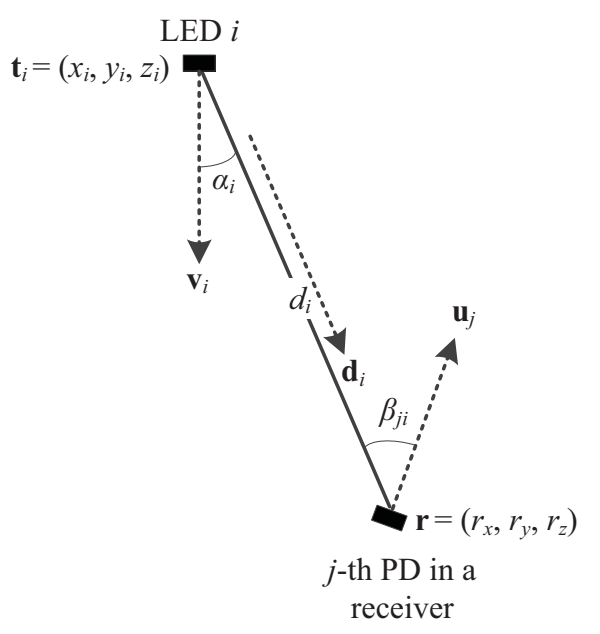

Fig. 1. The geometry of a line-of-sight (LoS) link between the $i$-th LED and the $j$-th PD in a $M \times N$ MIMO-VLC system.

Section V.

\section{SYSTEM MODEL}

In this paper, we consider an indoor point-to-point MIMOVLC system where $M$ LED arrays are deployed at the ceiling at 3-dimensional position $\mathbf{t}_{i} \in \mathbb{R}^{3}$ for $i=1,2, \ldots, M$ and communicate with the receiver equipped with $N$ PDs at position $\mathbf{r} \in \mathbb{R}^{3}$. For simplicity, we here assume that the transmission distance from each LED array to PDs is much larger than the distance between PDs which will be negligible for calculating channel matrix. Therefore, as shown in Fig. 1, the transmission distance from each LED array to receiver can be given by $d_{i}=\left\|\mathbf{r}-\mathbf{t}_{i}\right\|$ and the unit direction vector is $\mathbf{d}_{i}=\left(\mathbf{r}-\mathbf{t}_{i}\right) / d_{i}$. We also assume that each LED and receiver communicate through the line-of-sight (LoS) link without reflections [3].

\section{A. Transmitter}

The intensity modulation scheme is utilized at each LED array where the input electric signal $s_{i}$ for $i=1,2, \ldots, M$ is modulated into each LED array and then converted into light intensity signal. Each LED is DC-biased to drive in its linear dynamic operating range [18], [19] which means that the LED drive current signal $s_{i}=x_{i}+I_{D C}$, where $x_{i}$ is the information-bearing signal and $I_{D C}$ is the DC-bias. We here note that we impose the same DC-bias level at every LED array in order to maintain the uniform illumination level over the room area. Each LED array emits Lambertian radiated light centered in the direction of unit vector $\mathbf{v}_{i}$ for $i=1,2, \ldots, M$, which is given by

$$
R_{i}=\frac{m+1}{2 \pi} \cos ^{m}\left(\alpha_{i}\right),
$$

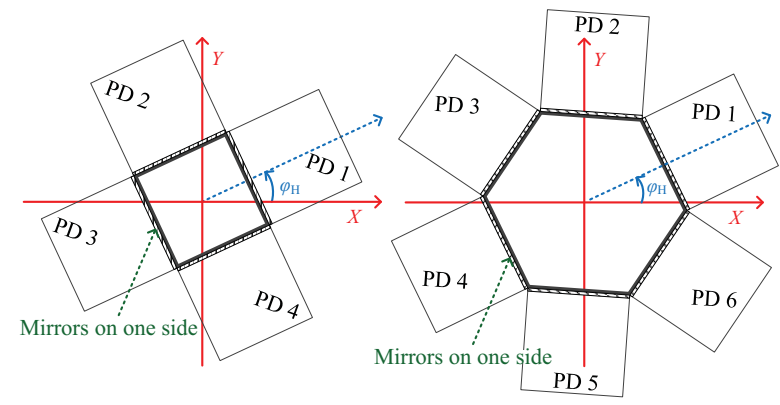

(a) Top view of MADR for examples of $N=4$ and $N=6$ (Hatching lines in the figure symbolize a mirror.)

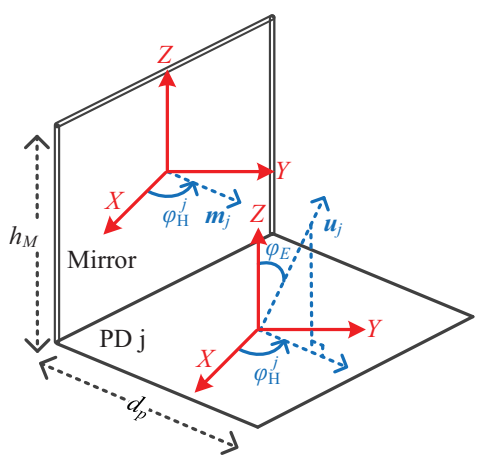

(b) A unit of the $j$-th mirror-PD pair

Fig. 2. The structure of the proposed mirror-aided angle diversity receiver (MADR).

where $\alpha_{i}$ is the irradiance angle at the $i$-th LED array to the receiver which is computed as $\cos \left(\alpha_{i}\right)=\mathbf{d}_{i}^{T} \mathbf{v}_{i} \cdot m$ is the Lambertian emission order which is given by [20]

$$
m=\frac{-\ln 2}{\ln \left(\cos \Phi_{1 / 2}\right)},
$$

where $\Phi_{1 / 2}$ denotes the semi-angle at half-power of light emission in the LED.

\section{B. Proposed Receiver}

In this paper, we propose a mirror-aided angle diversity non-imaging receiver (MADR) in a general form as shown in Fig. 2. The key features of the proposed receiver design are 1) tilting the azimuth and elevation angles of each PD to represent the different incident directions and 2) alleviating the channel correlation between the different incident angles by receiving the reflected light or blocking by a mirror. For ease of explanation, we assume that the receiver is placed horizontally. The pyramid-structured ADR in [12] can fulfil the design concept in which each PD is oriented into the separable incident direction. The PDs are deployed uniformly on the horizontal plane $(x-y$ plane) in order to orient the spatial direction separated by $\frac{2 \pi}{N}$ [rad]. As shown in Fig. 2a, the azimuth angle of the $j$-th PD is given by $\phi_{H}^{j}=\phi_{H}+\frac{2(j-1) \pi}{N}$ for $j=1,2, \ldots, N$. As shown in Fig. $2 \mathrm{~b}$, the elevation angle for the $j$-th PD is given by $\phi_{E}$ which is equal for all the PDs. 
Based on the azimuth and elevation angles, the normal vector, $\mathbf{u}_{j}$, orthonormal to the $j$-th PD plane can be computed as

$$
\mathbf{u}_{j}=\mathbf{R}_{z}\left(\phi_{H}^{j}\right) \mathbf{R}_{y}\left(\phi_{E}\right) \mathbf{e}_{3},
$$

where $\mathbf{R}_{z}(\vartheta)$ and $\mathbf{R}_{y}(\vartheta)$ are the basic rotation matrices rotating vectors by an angle $\vartheta$ about $z$ and $y$-axis in three dimensions which are given by

$\mathbf{R}_{z}(\vartheta)=\left[\begin{array}{ccc}\cos \vartheta & -\sin \vartheta & 0 \\ \sin \vartheta & \cos \vartheta & 0 \\ 0 & 0 & 1\end{array}\right], \mathbf{R}_{y}(\vartheta)=\left[\begin{array}{ccc}\cos \vartheta & 0 & \sin \vartheta \\ 0 & 1 & 0 \\ -\sin \vartheta & 0 & \cos \vartheta\end{array}\right]$ and $\mathbf{e}_{n}$ is the basis vector with all zero elements excepting only the $n$-th element equal to one. As shown in Fig. $2 \mathrm{~b}$, the one-sided mirror is placed vertically from the receiver plane in order to reflect the light from the horizontal incident angle $\phi_{H}^{j}$ into the $j$-th PD plane, while to block the light from other horizontal angle partially or perfectly. Therefore, the normal vector of mirror orthonormal to the mirror plane is given by $\mathbf{m}_{j}=\mathbf{R}_{z}\left(\phi_{H}^{j}\right) \mathbf{e}_{1}$. We assume that the active area of each PD plane is $A=d_{p}^{2}$ in the shape of square with the length of one side equal to $d_{p}$ and the height of mirror is defined as $h_{M}$.

\section{Symbol Space Representation}

At the proposed receiver, each PD directly detects electrical current signal $y_{j}$ for $j=1,2, \ldots, N$ proportionally to the intensity of the received signal. After removing the DC-bias, the received signal can be represented in vector space as

$$
\mathbf{y}=\mathbf{H x}+\mathbf{n},
$$

where $\mathbf{n} \in \mathbb{R}^{N \times 1}$ denotes independent and identically distributed (i.i.d.) additive white Gaussian noise (AWGN) vectors with zero mean and variance $\sigma_{n}^{2} \mathbf{I}_{N}{ }^{1} \mathbf{H} \in \mathbb{R}^{N \times M}$ is the $N \times M$ channel matrix whose element $h_{j i}$ represents the channel gain between the $i$-th LED and the $j$-th PD. In the following sections, we calculate the channel matrix of aforementioned MIMO-VLC systems with the proposed nonimaging receiver and evaluate the performance of achievable rate.

\section{Channel Matrix of Proposed MADR}

In order to calculate the channel matrix of proposed MADR, we should know how deploying the mirror affects in the channel gain of elements in $\mathbf{H}$. We first calculate the channel gain of conventional non-imaging receiver, ADR in this case, by assuming no mirror deployment. Then, we add or subtract the amount of channel gain which is changed by cases due to the reception of reflected light or blockage of LoS light onto each PD plane by a mirror.

The channel gain of the conventional non-imaging receiver without a mirror can be easily computed as [20]. As shown in Fig. $1, \beta_{j i}$ is defined as the incident angle at the $j$-th PD with respect to the $i$-th LED array which is computed as $\cos \left(\beta_{j i}\right)=$

\footnotetext{
${ }^{1}$ We note that the shot and thermal noise in the receiver's electric domain are generally modeled as AWGN.
}
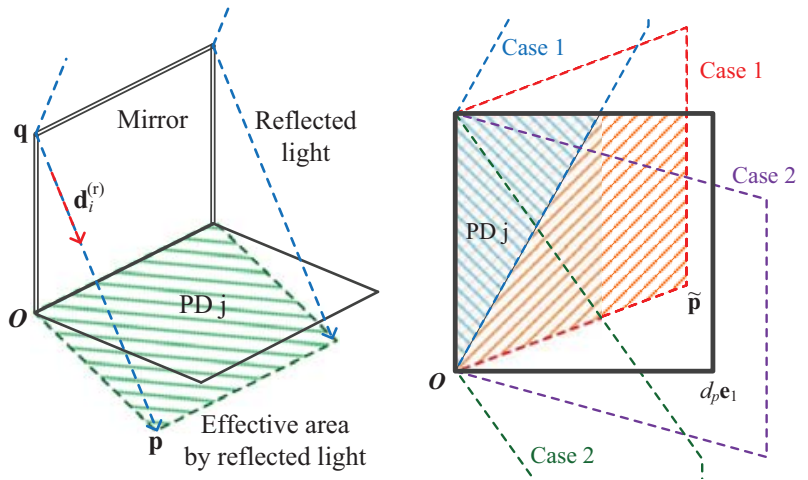

(a) Example of reflected light and its effective active area on PD plane.

b) Two different cases of effective active area affected by a mirror. (Hatching lines in the figure symbolize an effective area for case 1.)

Fig. 3. The reception of the reflected light in the proposed mirror-aided angle diversity receiver (MADR).

$-\mathbf{d}_{i}^{T} \mathbf{u}_{j}$. The channel gain from the $i$-th LED array to the $j$-th PD without a mirror is given by [20]

$$
h_{j i}^{(o)}= \begin{cases}\frac{R_{i} A}{d_{i}^{2}} \cos ^{m}\left(\alpha_{i}\right) \cos ^{k}\left(\beta_{j i}\right), & \beta_{j i}<\Psi_{c} \\ 0, & \beta_{j i}>\Psi_{c}\end{cases}
$$

where $\Psi_{c}$ and $k$ are the field-of-view (FoV) and its coefficient of the PD. ${ }^{2}$

As shown in Fig. 3a, the mirror influences the amount of intensity received at each PD in a unit. When the light is reflected by a mirror, the amount of intensity might be received at the PD. On the other hand, if the light is blocked by a mirror, it makes shade in the active area of PD plane and decreases the received intensity. By checking the correlation between the normal vector of mirror, $\mathbf{m}_{j}$, and the direction vector of light at a mirror with respect to the LED array, $-\mathbf{d}_{i}$, we can see whether the light is reflected or blocked by a mirror. Defining $\rho_{j i}=-\mathbf{m}_{j}^{T} \mathbf{d}_{i}, \rho_{j i}>0$ means that the light is reflected by a mirror but $\rho_{j i}<0$ means that the light is blocked. We here calculate the increasing amount of reflected light intensity received at the $j$-th PD from the $i$-th LED array in detail.

In order to calculate the effective active area of PD affected by reflected light, we find the point $\mathbf{p}$ of the light reflected at the edge of mirror $\mathbf{q}$ which is intersecting the plane where the PD is placed on. As shown in Fig. 3a, without loss of generality, we assume the origin of the cartesian coordinate $O$ at the edge of mirror below the point $\mathbf{q}$ which means $\mathbf{q}=\left[\begin{array}{lll}0 & 0 & h_{M}\end{array}\right]^{T}$. The direction vector of the reflected light can be computed as $\mathbf{d}_{i}^{(r)}=\mathbf{d}_{i}+2 \rho_{j i} \mathbf{m}_{j}$. The intersection point $\mathbf{p}$ is on the line $\mathbf{p}=\mathbf{q}+t \mathbf{d}_{i}^{(r)}$ for any $t$ and the plane $\mathbf{u}_{j}^{T} \mathbf{p}=0$. From these two equations, we can find

\footnotetext{
${ }^{2}$ We assume that the specification of all the PDs are the same.
} 
$t=-\frac{\mathbf{u}_{j}^{T} \mathbf{q}}{\mathbf{u}_{j}^{T} \mathbf{d}_{i}^{(r)}}$. Therefore, the intersection point is given by

$$
\mathbf{p}=\mathbf{q}-\frac{\mathbf{u}_{j}^{T} \mathbf{q}}{\mathbf{u}_{j}^{T} \mathbf{d}_{i}^{(r)}} \mathbf{d}_{i}^{(r)}
$$

Depending on the point $\mathbf{p}$ located on the plane, two different effective active area of PD plane can be taken into consideration as shown in Fig. 3b. The effective active area is in a form of vertically flipped right triangle or rectangular for case 1 and rectangular or right triangle for case 2 , respectively. Without loss of generality, it is convenient to distinguish two cases if we rotate the coordinate back according to the elevation and azimuth angles of the $j$-th PD. Letting the rotated point $\tilde{\mathbf{p}}=\mathbf{R}_{y}\left(-\phi_{E}\right) \mathbf{R}_{z}\left(-\phi_{H}^{j}\right) \mathbf{p}=\left[\begin{array}{lll}\tilde{p}_{x} & \tilde{p}_{y} & \tilde{p}_{z}\end{array}\right]^{T}$, the $j$-th PD and $\tilde{\mathbf{p}}$ are now located on the $x-y$ plane as shown in Fig. $3 \mathrm{~b}$. The four points at the edges of the $j$-th PD are on the origin, $d_{p} \mathbf{e}_{1}$, $d_{p} \mathbf{e}_{2}$, and $d_{p}\left(\mathbf{e}_{1}+\mathbf{e}_{2}\right)$, respectively. For $\tilde{p}_{x}<0$, the reflected light cannot reach the PD plane and the effective active area becomes zero. For $\tilde{p}_{x}>0$, the case 1 occurs when $\frac{\tilde{p}_{y}}{\tilde{p}_{x}}>0$ and vice versa. For case 1 , depending on the position of $\left(\tilde{p}_{x}, \tilde{p}_{y}\right)$, the effective active area can be calculated by subtracting the left triangle area where is not affected by reflected light from the rectangular area including the effective active area and this triangle area. Therefore, for $\frac{\tilde{p}_{y}}{\tilde{p}_{x}}>0$, the effective active area can be computed as

$$
\begin{aligned}
A_{j i}^{\mathrm{eff}} & =d_{p} \min \left(d_{p},\left|\tilde{p}_{x}\right|, d_{p}\left|\frac{\tilde{p}_{x}}{\tilde{p}_{y}}\right|\right) \\
& -\frac{1}{2} \min \left(d_{p},\left|\tilde{p}_{y}\right|, d_{p}\left|\frac{\tilde{p}_{y}}{\tilde{p}_{x}}\right|\right) \min \left(d_{p},\left|\tilde{p}_{x}\right|, d_{p}\left|\frac{\tilde{p}_{x}}{\tilde{p}_{y}}\right|\right) .
\end{aligned}
$$

We note that the absolute in $\tilde{p}_{x}$ and $\tilde{p}_{x}$ is required to unify the effective active area for other cases.

The case 2 is symmetric to the case 1 over $\frac{\tilde{p}_{y}}{\tilde{p}_{x}}=0$ and thus the effective active area for $\frac{\tilde{p}_{y}}{\tilde{p}_{x}}<0$ can be computed similarly to the case 1 by taking the absolute of $\tilde{p}_{y}$ as in (7). The effective channel gain received by the reflected light can be finally computed as

$h_{j i}^{\mathrm{eff}}= \begin{cases}\eta_{M} \frac{R_{i} A_{j i}^{\mathrm{eff}}}{d_{i}^{2}} \cos ^{m}\left(\alpha_{i}\right) \cos ^{k}\left(\beta_{j i}^{(r)}\right), & \beta_{j i}^{(r)}<\Psi_{c} \& \tilde{p}_{x}>0 \\ 0, & \text { otherwise }\end{cases}$

where $\eta_{M} \in[0,1]$ is the mirror's reflection coefficient which is equal to one for a perfect mirror and $\beta_{j i}^{(r)}$ is the incident angle at the $j$-th PD with respect to the reflected light of the $i$-th LED array which is computed as $\cos \left(\beta_{j i}^{(r)}\right)=-\mathbf{u}_{j}^{T} \mathbf{d}_{i}^{(r)} /\left\|\mathbf{d}_{i}^{(r)}\right\|$.

On the other hand, in order to calculate the effective active area of PD shaded by light blockage due to the mirror $\left(\rho_{j i}<\right.$ 0 ), we can calculate the effective channel gain similarly to that for the reflected light. For $\tilde{p}_{x}>0$, the only difference is to use the direction vector $\mathbf{d}_{i}$ instead of $\mathbf{d}_{i}^{(r)}$ since there is no reflection. For $\tilde{p}_{x}<0$, it means that the light propagation can not reach the PD plane regardless of the existence of a

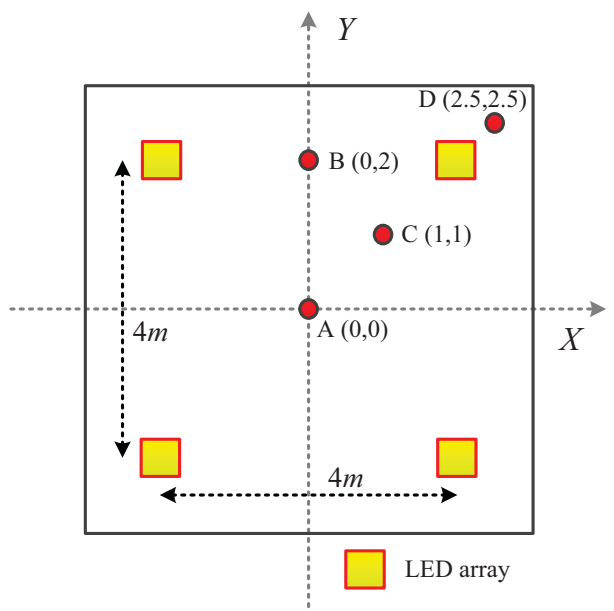

Fig. 4. The geometry of transmitter and receiver for a $4 \times 4$ MIMO-VLC system in indoor scenario (top view).

mirror. Then, the effective channel gain decreased by blocking the light can be computed as

$$
h_{j i}^{\mathrm{eff}}=\left\{\begin{array}{ll}
-\frac{R_{i} A_{j i}^{\mathrm{eff}}}{d_{i}^{2}} \cos ^{m}\left(\alpha_{i}\right) \cos ^{k}\left(\beta_{j i}\right), & \beta_{j i}<\Psi_{c} \& \tilde{p}_{x}>0 \\
0, & \text { otherwise }
\end{array} .\right.
$$

Finally, the element of channel matrix $\mathbf{H}$ for the proposed MADR in $N \times M$ MIMO-VLC systems is given by

$$
h_{j i}=h_{j i}^{(o)}+h_{j i}^{\mathrm{eff}} .
$$

Here we assume that the LED efficiency and PD responsivity are omitted to be unity without loss of generality but we can consider them according to the specification of off-the-shelf products.

\section{NumeriCAL RESUlts}

In our performance evaluation, we consider the channel capacity of the MIMO-VLC system with the known channel state information (CSI) at the receiver side only. When the information-bearing transmit signal is constrained on $\mathbb{E}\left[\|\mathbf{x}\|^{2}\right]=P$. Under equal power allocation, the channel capacity can be computed as ${ }^{3}$

$$
R=\frac{1}{2} \log _{2} \operatorname{det}\left|\mathbf{I}+\rho \mathbf{H} \mathbf{H}^{T}\right|,
$$

where $\rho \triangleq \frac{P}{\sigma^{2}}$ is the average electrical signal-to-noise ratio (SNR) per LED.

For the performance analysis, as shown in Fig. 4, we consider a spacious room where the nearest LED arrays are 4 meter away from each other with the height of 3 meter $(z=3)$

${ }^{3}$ This is the capacity formula in the classical MIMO system. The channel capacity bounds in an optical intensity channel are obtained in [21, Eq. (7.43)]. We note that the above capacity formula is enough to verify the performance of proposed receivers because 1) it is used in the previous literature (See [12] and references therein.); and 2) it is in the same form as the achievable rate formula of constant power and continuous rate adaptation optical MIMO systems [22, Eq. (24)-(25)]. 


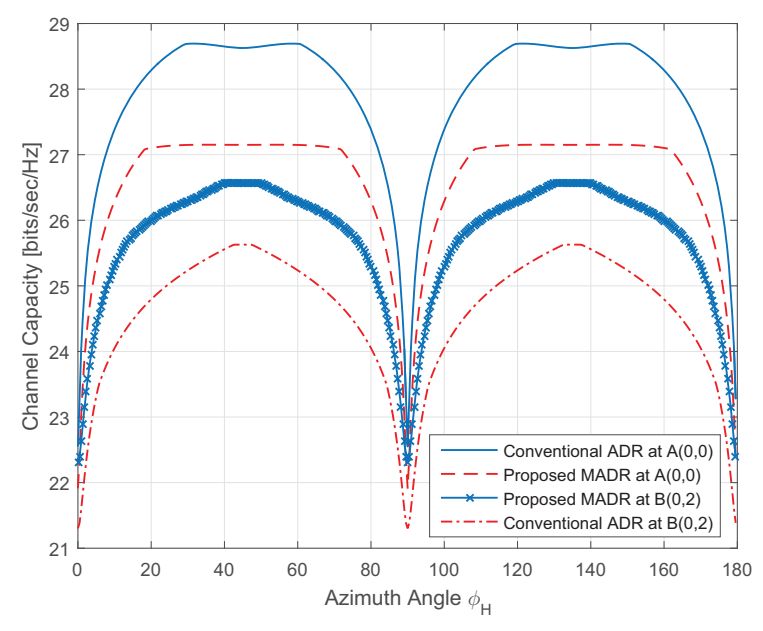

Fig. 5. The channel capacity comparison in a $4 \times 4$ MIMO-VLC system with respect to the azimuth angle of receiver at positions $A(0,0)$ and $B(0,2)$.

and different positions of receiver ( $A$ to $D$ ) with the height of 1.2 meter $(z=1.2)$. Here we assume that the receiver is placed horizontally to directly face the LED arrays. We evaluated the channel capacity of $4 \times 4$ MIMO-VLC systems with conventional angle diversity receiver (ADR) and our proposed mirror-aided ADR (MADR). Therefore, the PDs are rotated horizontally every $\frac{\pi}{2}$. The elevation angle of PD plane is chosen to maximize the channel capacity at each position, i.e., $\phi_{E}^{\star}=\arg \max _{\phi_{E}} R$. The detailed system parameters for simulation is listed in Table I.

TABLE I

SYSTEM PARAMETERS FOR SIMULATION

\begin{tabular}{|c|c|c|}
\hline & Parameters & Values \\
\hline LED & $\begin{array}{c}\text { Locations at }\left(\mathbf{t}_{i}\right) \\
\text { Direction vector of irradiation }\left(\mathbf{v}_{i}\right) \\
\text { Semi-angle at a half power }\left(\Phi_{1 / 2}\right) \\
\text { Lambertian emission order }(m) \\
\text { Electrical SNR per LED }(\rho)\end{array}$ & $\begin{array}{c}( \pm 2, \pm 2,3)^{\dagger} \\
{\left[\begin{array}{c}0 \\
0-1\end{array}\right]^{T}} \\
60^{\circ} \\
1 \\
160 \mathrm{~dB}\end{array}$ \\
\hline Receiver & $\begin{array}{c}\text { Locations } \\
\text { Receiver field-of-view (FoV) } \\
\text { FoV coefficient }(k) \\
\text { Active area of PD }(A) \\
\text { Length of one side at PD plane }\left(d_{p}\right) \\
\text { Height of mirror }\left(h_{M}\right) \\
\text { Reflection coefficient of mirror }\left(\eta_{M}\right)\end{array}$ & $\begin{array}{l}A \text { to } D \\
180^{\circ} \\
1 \\
1 \mathrm{~cm}^{2} \\
1 \mathrm{~cm} \\
1 \mathrm{~cm} \\
0.9 \mathrm{~cm}\end{array}$ \\
\hline
\end{tabular}

${ }^{\dagger}$ The unit of distance is meter $(\mathrm{m})$.

Fig 5 and Fig. 6 present the channel capacity of a $4 \times 4$ MIMO-VLC system with two different receivers with respect to different receiver positions. According to the varying azimuth angle, the receiver performance is symmetric at every $\frac{\pi}{4}$ since the receiver structure is also symmetric every $\frac{\pi}{4}$. From the figures, we can see that our proposed MADR can improve the channel capacity at positions $A, B$, and $C$. This means that the proposed receiver can further improve the capacity over ADR when the channel correlation is dominant factor to degrade the channel capacity in this region where is relatively

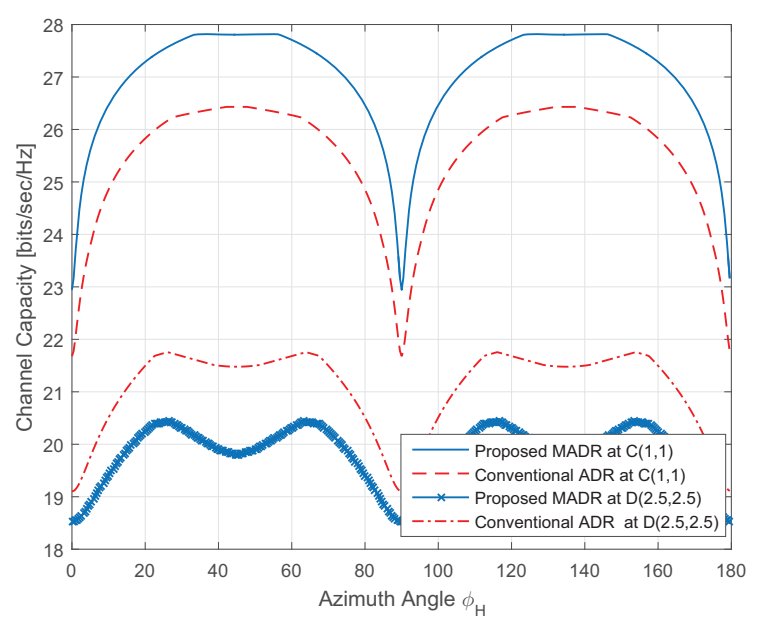

Fig. 6. The channel capacity comparison in a $4 \times 4$ MIMO-VLC system with respect to the azimuth angle of receiver at positions $C(1,1)$ and $D(2.5,2.5)$.

close to the room center. For positions $A(0,0)$ and $C(1,1)$, the channel capacity is maximized at $\phi_{H}=\frac{\pi}{4}, \frac{3 \pi}{4}$ since each PD can face each LED array respectively in azimuthal domain and thus the channel is less correlated, while minimized at $\phi_{H}=0, \frac{\pi}{2}$ since two PDs are equally receiving the light from each LED array and thus channel matrix are highly correlated. For position $D(2.5,2.5)$, the channel capacity is not maximized at $\phi_{H}=\frac{\pi}{4}, \frac{3 \pi}{4}$. The strongest link from the nearest LED array is dominating the performance and increasing the channel gain over all PDs from the nearest LED array is more significant at position $D(2.5,2.5)$. However, the proposed MADR is still blocking the reception of the light from the nearest LED to some PDs and this is the reason why the proposed MADR performs worse than conventional ADR at this position. Although the receiver is placed horizontally, we can compensate for the performance loss at position $D$ by tilting the receiver to the nearest LED array in order to alleviate the blockage due to a mirror.

In Table II, we show the average channel capacity over the azimuth angle at different positions. We can see from the table that the proposed MADR can provide around 1.0-1.5 bps/Hz of capacity gain on average at positions $A, B$, and $C$ but lose nearly $1.3 \mathrm{bps} / \mathrm{Hz}$ of gain at position $D$.

TABLE II

AVERAGE CHANNEL CAPACITY AT DIFFERENT POSITIONS [BPS/Hz]

\begin{tabular}{c|c|c}
\hline \hline Position & MADR & ADR \\
\hline$A(0,0)$ & 27.88 & 26.58 \\
$B(0,2)$ & 25.72 & 24.61 \\
$C(1,1)$ & 26.98 & 25.48 \\
$D(2.5,2.5)$ & 19.81 & 21.07 \\
\hline \hline
\end{tabular}

\section{CONCLUSION}

In this paper, we proposed new non-imaging receiver design incorporating the advantage of deploying a mirror onto 
conventional angle diversity receiver. The channel gain of proposed MADR is derived based on the transmitter/receiver geometry and the receiver structure characterized by azimuth and elevation angles of PDs and mirrors. The numerical results show that the proposed scheme can further improve the channel capacity in the area where the channel correlation can greatly affect the channel capacity. On the other hand, when the channel gains from the nearest LED array are more dominant than the channel correlation, mirror can reduce the desired channel gains by blocking the light from the nearest LED array and therefore tilting the entire receiver plane to the nearest LED array is required to avoid the blockage.

\section{REFERENCES}

[1] D. C. O'Brien, L. Zeng, H. L. Minh, G. Faulkner, J. W. Walewski, and S. Randel, "Visible light communications: challenges and possibilities," in Proc. IEEE Personal, Indoor and Mobile Radio Communications (PIMRC 2008), Cannes, France, Sep. 2008.

[2] H. Haas, L. Yin, Y. Wang, and C. Chen, "What is LiFi?" J. Lightw. Technol., vol. 34, no. 6, pp. 1533-1544, Mar. 2016.

[3] L. Zeng, D. O’Brien, H. Minh, G. Faulkner, K. Lee, D. Jung, Y. Oh, and E. T. Won, "High data rate multiple input multiple output (MIMO) optical wireless communications using white LED lighting," IEEE J. Sel. Areas Commun., vol. 27, no. 9, pp. 1654-1662, Dec. 2009.

[4] Z. Chen and H. Haas, "Space division multiple access in visible light communications," in Proc. IEEE Int. Conf. Commun. (ICC), London, UK, Jun. 2015, pp. 5115-5119.

[5] L. Yin, X. Wu, and H. Haas, "On the performance of non-orthogonal multiple access in visible light communication," in Proc. IEEE Annu. Symp. Personal, Indoor and Mobile Radio Commun. (PIMRC), Hong Kong, Sep. 2015, pp. 1376-1381.

[6] A. Burton, H. L. Minh, Z. Ghassemlooy, E. Bentely, and C. Botella, "Experimental demonstration of 50-Mb/s visible light communications using $4 \times 4$ MIMO," IEEE Photon. Technol. Lett., vol. 26, no. 9, pp. 945-948, May 2014

[7] Y. Wang and N. Chi, "Demonstration of high-speed $2 \times 2$ nonimaging MIMO Nyquist single carrier visible light communication with frequency domain equalization,", J. Lightw. Technol., vol. 32, no. 11, pp. 2087-2093, Jun. 2014.

[8] A. H. Azhar, T. Tran, and D. O'Brien, "A Gigabit/s indoor wireless transmission using MIMO-OFDM visible-light communications," IEEE Photon. Technol. Lett., vol. 25, no. 2, pp. 171-174, Jan. 2013.

[9] T. Q. Wang, Y. A. Sekercioglu, and J. Amstrong, "Analysis of an optical wireless receiver using a hemispherical lens with application in MIMO visible light communications," J. Lightw. Technol., vol. 31, no. 11, pp. 1744-1754, Jun. 2013.

[10] T. Chen, L. Liu, B. Tu, Z. Zheng, and W. Hu, "High-spatial-diversity imaging receiver using fisheye lens for indoor MIMO VLCs," IEEE Photon. Technol. Lett., vol. 26, no. 22, pp. 2260-2263, Nov. 2014

[11] T. Fath and H. Haas, "Performance comparison of MIMO techniques for optical wireless communications in indoor environments," IEEE Trans. Commun., vol. 61, no. 2, pp. 733-742, Feb. 2013.

[12] A. Nuwanpriya, S.-W. Ho, and C. S. Chen, "Indoor MIMO visible light communications: Novel angle diversity receivers for mobile users," IEEE J. Sel. Areas Commun., vol. 33, no. 9, pp. 1780-1792, Sep. 2015.

[13] T. Q. Wang, R. J. Green, and J. Armstrong, "MIMO optical wireless communications using ACO-OFDM and a prism-array receiver," IEEE J. Sel. Areas Commun., vol. 33, no. 9, pp. 1959-1971, Sep. 2015.

[14] C. He, T. Q. Wang, and J. Armstrong, "MIMO optical wireless receiver using photodetectors with different field of view," in Proc. IEEE Vehicular Technol. Conf. (VTC), Glasgow, Scotland, May 2015.

[15] K.-H. Park, H. M. Oubei, W. G. Alheadary, B. S. Ooi, and M.-S. Alouini, "A novel mirror-aided non-imaging receiver for indoor $2 \times 2$ MIMOvisible light communication systems," IEEE Trans. Wireless Commun., vol. 16, no. 9, pp. 5630-5643, Sep. 2017.

[16] P. Fahamuel, J. Thomson, and H. Haas, "Improved indoor VLC MIMO channel capacity using mobile receiver with angular diversity detectors," in Proc. IEEE Global Commun. Conf. Workshop, Austin, TX, USA, Dec. 2014.
[17] M. Yasir, S.-W. Ho, and B. N. Vellambi, "Indoor position tracking using multiple optical receivers," J. Lightw. Technol., vol. 34, no. 4, pp. $1166-$ 1176, Feb. 2016.

[18] H. Elgala, R. Mesleh, and H. Haas, "An LED model for intensitymodulated optical communication systems," IEEE Photon. Technol. Lett., vol. 22, no. 11, pp. 835-837, Jun. 2010.

[19] S. Dimitrov and H. Haas, "Information rate of OFDM-based optical wireless communication systems with nonlinear distortion," J. Lightw. Technol., vol. 31, no. 6, pp. 918-929, Mar. 2013.

[20] J. M. Kahn and J. R. Barry, "Wireless infrared communications," Proc. IEEE, vol. 85, no. 2, pp. 265-298, Feb. 1997.

[21] S. Arnon, J. Barry, G. Karagiannidis, R. Schober, and M. Uysal, Advanced Optical Wireless Communication Systems. Cambridge University Press, 2013.

[22] K.-H. Park, Y.-C. Ko, and M.-S. Alouini, "On the power and offset allocation for rate adaptation of spatial multiplexing in optical wireless MIMO channels," IEEE Trans. Commun., vol. 61, no. 4, pp. 1535-1543, Apr. 2013. 